\title{
MAPPING 3D TEXTILES ONTO THEIR MODELS
}

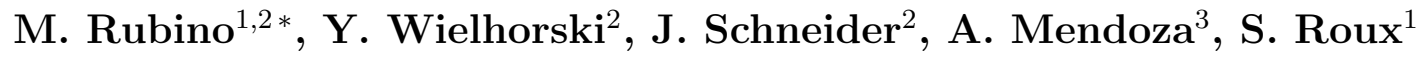 \\ ${ }^{1}$ LMT (ENS Paris-Saclay/CNRS/Univ. Paris-Saclay), 4, Avenue des Sciences, 91192 \\ Gif-sur-Yvette, France \\ 2 Safran Aircraft Engines, Rond Point Réné Ravaud-Réau, 77550 Moissy-Cramayel, France \\ ${ }^{3}$ Safran Tech, Rue des Jeunes Bois, 78772 Magny les Hameaux, France \\ *marcello.rubino@ens-paris-saclay.fr
}

The objective of the present study is to propose an automatic method for aligning the distortions of yarn layers in a 3D woven textile. The analysis is carried on the tomographic image of the preform molded to its final shape. A suited Gaussian filter erases either the warp or the weft structure so as to obtain a stratified structure, which is mapped onto a perfect fabric geometry characterized by a constant spacing of the yarn planes, as it would have been ideally produced just out of the loom.

The registration procedure is conducted in the spirit of Digital Volume Correlation (DVC). The "ideal image" may be constructed with different levels of sophistication that incorporate more or less information about yarns, and their spacing. The registration of the image with its ideal model is thus coined model-based correlation method (MDIC). It allows a more faithful description of the effect of moving two yarn plane closer or farther apart, which for tomography violates the usual conservation of gray levels. The algorithm adjusts each yarn surface along the mean surface normal and is discretized along a regular grid whose step correspond to the typical yarn spacing. Thus the problem reduces to a set of one-dimensional image correlation problems, that make the numerical resolution quite fast. The identity of the single yarn surfaces is accounted for in the model image, as each parallel yarn plane is modeled as having a Gaussian profile whose amplitude corresponds to the microstructural density of yarn columns. The linearly interpolated (scalar) displacement field is applied to the raw volumetric image in both transverse directions, hence obtaining the full correction of the textile, as shown in Figure 1, providing perfectly vertical yarn planes with a constant spacing both along warp and weft orders.

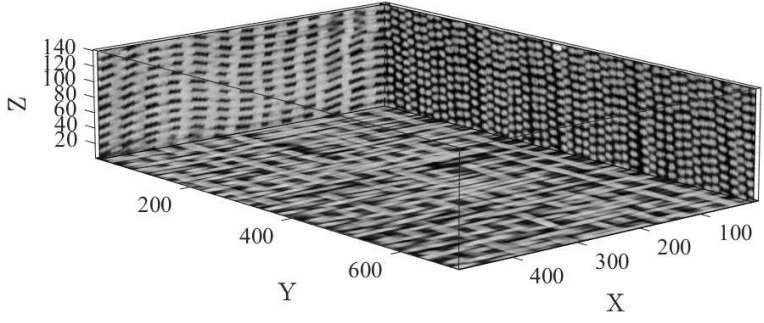

(a)

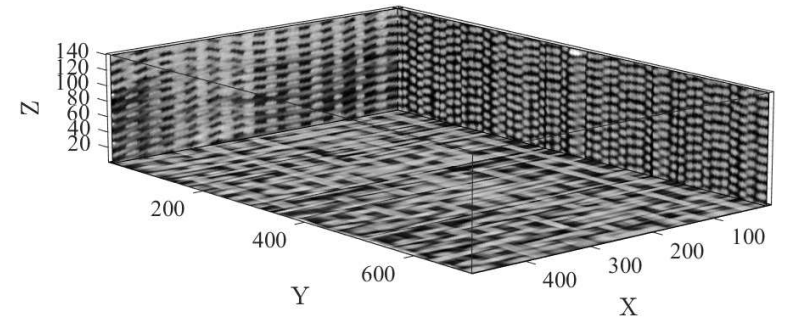

(b)

Figure 1: Correction of a 3D woven specimen: (a) raw configuration; (b) aligned configuration 